\title{
The case of optimal control with exceptional role of the conditions of transversality
}

\begin{abstract}
This research considered solving the optimal control problem of spacecraft (as solid body) when the conditions of transversality have key significance. It is shown that the assumed criterion of optimality guarantees motion of spacecraft with energy not exceeding the required value. Topicality of article is caused by fact that on concrete example, the conditions of transversality are demonstrated to be very important mathematical instrument (even the only) for finding the main properties, laws and key characteristics (parameters, constants, integrals of motion) of optimal solution of control problem.
\end{abstract}

Keywords: optimal control, criterion of optimality, maximum principle, conditions of transversality, controlling functions
Volume 3 Issue 3 - 2019

\section{Levskii}

Khrunichev State scientific \& production Space Center, Russia

Correspondence: MV Levskii, Khrunichev State scientific \& production Space Center, Russia, Tel (499) 277-37-I6, Emaildp940@mail.ru

Received: September 25, 2017 | Published: October 17, 2017

\section{Introduction}

Its investigate motion of spacecraft (as solid body) relative to centre of mass was investigated in this research work. Spacecraft attitude is described by the quaternion $\Lambda$ (it give position of the body axes relative to inertial coordinate system) and the vector $\omega$ of absolute angular velocity. Then, equation of motion ${ }^{1}$

$$
2 \dot{\Lambda}=\Lambda \circ \omega
$$

(It is assumed that $\|\Lambda(0)\|=1$ ). In order to estimate the efficiency of control, the functional to be optimized is introduced as presented in equation 2 .

$$
G=\int_{0}^{T}\left(a_{1}\left(J_{1} \omega_{1}^{2}+J_{2} \omega_{2}^{2}+J_{3} \omega_{3}^{2}\right)+a_{2}\right) d t
$$

Where $J_{i}$ are the spacecraft central principal moment of inertia; $\omega_{i}$ are the components of vector $\omega(i=\overline{1,3}) ; a_{1}=$ const $>0, a_{2}=$ const $>0$. Let solve following problem of control: take the spacecraft from initial attitude $\Lambda_{\text {in }}$ into final attitude $\Lambda_{f}$ obeying Eq. (1) so as to minimize integral (2) (the time $\mathrm{T}$ is not given). The taken criterion of optimality combines (in given proportion) the time and integral of energy to be expended for slew maneuver. Aspects of finding economical control are topical now.

For solving the formulated problem, use Pontryagin's maximum principle $^{2}$ and the universal variables ${ }^{3}$ (because the optimized functional does not include positional coordinates). The assumed integral (2) does not include the torques $M_{i}$; the sought-for function $\omega(t)$ is piecewise continuous function of time. For our optimization problem, the Hamiltonian is

$$
H=r_{1} \omega_{1}+r_{1} \omega+r_{1} \omega-a_{1}\left(J_{1} \omega_{1}^{2}+J_{2} \omega_{2}^{2}+J_{3} \omega_{3}^{2}\right)-a_{2},
$$

Where $r_{i}$ are universal variables (as the components of vector $\mathrm{r}$ ) satisfying equations ${ }^{3}$

$$
r_{1}=\omega_{3} r_{2}-\omega_{2} r_{3}, r_{2}=\omega_{1} r_{3}-\omega_{3} r, r_{3}=\omega_{2} r_{1}-\omega_{12} r_{2}
$$

The Hamiltonian $H$ is written, ignoring the constraint $\|\Lambda\|=1$ since $\|\Lambda(t)\|=1$ under any $\omega(t)$ for Eq. (1) (of course $\left\|\Lambda_{\text {in }}\right\|=\left\|\Lambda_{f}\right\|=1$ ). Optimal function $\mathrm{r}(t)$ is computed by the quaternion $\Lambda(t)::^{1,3}$

$$
r=\tilde{\Lambda}{ }^{\circ} C_{E} \circ \Lambda \text {, where } c E=\text { const }=\Lambda_{\text {in }} \circ r(0) \circ \tilde{\Lambda}_{i n}
$$

For the vector $r$ of universal variables $|r|=$ const $\neq 0$. The function $\mathrm{H}$ is maximal if the relations

$$
\omega_{i}=r_{i} / 2 a_{1} J
$$

are satisfied. As is known, the functions $r_{i}$ and $\omega_{i}$ should satisfy the conditions of transversality which are $\mathrm{r}(0) \neq 0, \mathrm{r}(T) \neq 0$ (since left and right endpoints of the trajectory $\Lambda(t)$ are fixed) and $\mathrm{H}=0$ because the maneuver end time $\mathrm{T}$ is not fixed and the Hamiltonian $\mathrm{H}$ is independent of time in explicit form. After substitution Eq.(4) in expression for $\mathrm{H}$ and the requirement $\mathrm{H}=0$, obtain the equation

$$
\left(r_{1}^{2} / J_{1}+r_{2}^{2} / J_{2}+r_{3}^{2} / J_{3}\right) / 4 a_{1}-a_{2}=0
$$

Through which have following key properties of the controlled motion:

$$
\begin{aligned}
& r_{1}^{2} / J_{1}+r_{2}^{2} / J_{2}+r_{3}^{2} / J_{3}=\text { const }=4 a_{1} a_{2}, \\
& \mathrm{~J}_{1} \omega_{1}^{2}+\mathrm{J}_{2} \omega_{2}^{2}+\mathrm{J}_{3} \omega_{3}^{2}=\text { const }, \\
& J_{1}^{2} \omega_{1}^{2}+J_{2}^{2} \omega_{2}^{2}+J_{3}^{2} \omega_{3}^{2}=\text { const }
\end{aligned}
$$

Last property follows directly from the demands (4) (they formalize condition of maximum for $\mathrm{H}$ ). The condition of transversality $\mathrm{H}=0$ takes place at each instant of time. ${ }^{4}$

The problem of optimal control is reduced to finding the solution to the system of differential equations (1), (3) under the condition that the control $\omega$ is chosen based on condition (4) with the simultaneous satisfaction of the condition of transversality $\mathrm{H}=0$ and

the boundary conditions $\Lambda(0)=\Lambda_{i n}, \Lambda(T)=\Lambda_{f}$ (the conditions of transversality $\mathrm{r}(0) \neq 0$ and $\mathrm{r}(\mathrm{T}) \neq 0$ in are satisfied automatically, as it follows from first equality (5) written for optimal motion). The system of differential equations (3) for the variables $r_{i}$, together with the requirement of maximizing the Hamiltonian $\mathrm{H}$ and the condition $\mathrm{H}=0$, provides the necessary optimality conditions. Reminding that the coefficients $a_{1} \neq 0$ and $a_{2} \neq 0$. If we take the ort then $p=r /|r|$ then $r_{0}=2 \sqrt{a_{1} a_{2}} / C ; E_{k}=a_{2} / 2 a_{1} ;|L|=\sqrt{a_{2} / a_{1}} / C$ where $r_{0}=|r|$; $C=\sqrt{P_{10}^{2} / J_{1}+P_{20}^{2} / J_{2}+P_{30}^{2} / J_{3}} ; p_{i 0}$ are the components of the vector 
$p_{0}=p(0) ; E_{k}$ is rotary energy; $\mathrm{L}$ is angular momentum;

$$
P_{1}^{2} / J_{1}+P_{2}^{2} / J_{2}+P_{3}^{2} / J_{3}=\text { const } \text {, since }|r|=\text { const. }
$$

The boundary value problem of the maximum principle is to determine such value of the vector $p_{0}$ at which the solution $\Lambda(t)$ of the motion equation (1) and differential equations (3) (with the simultaneous satisfying the equalities (4) at each instant of time) satisfies the maneuver conditions $\Lambda(0)=\Lambda_{i n}$ and $\Lambda(T)=\Lambda_{f}$ (the quantity $r_{0}$ is calculated unambiguously by $a_{1}, a$ and the coefficients $a_{1}, a_{2}$ ). Optimal vector $\mathrm{p}_{0}$ is determined only by the values $\Lambda_{i n}, \Lambda_{f}$ and $J_{1}, J_{2}, J_{3}$.

Punctual consecutive implementation of procedure of the maximum principle for dynamical problem of optimal slew maneuver (when $\omega(0)=\omega(T)=0$ and the control torque M is limited) show that maximal rotary energy $E_{k}$ is no more the ratio $a_{2} / 2 a_{1}$ for any instant of time $t \in[0, T]$ (independently of duration of acceleration and braking). I.e. always, during optimal rotation from the position $\Lambda_{\text {in }}$ into the position $a_{a}, a$ (in the sense of minimum (2)), rotary energy of spacecraft have restriction by known upper level determined by the coefficients $a_{1}, a_{2}$ of the minimized functional. If assume $a_{1}$ $=0.5$ and $a_{2}=E$ then optimization of motion program by criterion (2) give satisfaction of the inequality $J_{1} \omega_{1}^{2}+J_{2} \omega_{2}^{2}+J_{3} \omega_{3}^{2} \leq E_{k}$ for any instant of time, where $E_{a d}$ is admissible rotary energy.
In our variation problem, find the main properties, laws and key characteristics (parameters, constants, integrals of motion) of optimal solution of control problem using the conditions of transversality as very important and unique mathematical instrument. Chosen criterion of optimality guarantees motion of solid body with rotary energy not exceeding the required value.

\section{Acknowledgments}

No financial exists.

\section{Conflict of interest}

No conflict of interest exists.

\section{References}

1. Branets VN, Shmyglevskii IP. Use of quaternions in the problems of orientation of solid body. 1973.

2. Pontryagin LS, Boltyanskii VG, Gamkrelidze RV, et al. The mathematical theory of optimal processes. JSTOR. 1986;19(89):159-161.

3. Levskii MV. The use of universal variables in problems of optimal control concerning spacecrafts orientation. Mechatronics, automation, control. 2014.

4. Young LG. Lectures on the calculus of variations and optimal control theory. Canada: WB Saunders Company; 1969 